\title{
Forum
}

\section{La modélisation comme outil d'accompagnement}

\author{
Collectif ComMod*
}

\section{Posture}

Les recherches que nous menons (Encadré) portent sur la gestion des ressources renouvelables et de l'environnement. Elles nous mettent donc face à des objets d'étude complexes et dynamiques, qui sont aussi objets d'enjeux et d'actions multiples. D'où notre choix de faire des recherches dites «impliquées », c'est-à-dire qui reposent sur une double contrainte librement consentie : d'une part, des investissements théoriques et méthodologiques jugés par les pairs, mais issus de questions de terrain; d'autre part, des terrains choisis à la fois en fonction de leur intérêt méthodologique et de leur pertinence pour les usagers et les décideurs avec qui nous travaillons. Cette implication est utilisée comme moyen privilégié de tester et de remettre en cause les principes sur lesquels reposent les actions de terrain. Nous sommes donc aux prises avec un questionnement à la fois pragmatique et théorique. Cela implique la reconnaissance de l'incertitude dans la situation de décision et de l'existence de multiples points de vue légitimes, expertises scientifiques comprises. Ces différents points de vue méritent d'être pris en compte dans un processus itératif de compréhension, de confrontation et d'analyse. Nous avons

\footnotetext{
Auteur correspondant :

M. Étienne, etienne@avignon.inra.fr

* Companion Modelling. Les signataires de ce texte sont (par ordre alphabétique) : Martine Antona (économiste, Cirad), Patrick d'Aquino (géographe, Cirad), Sigrid Aubert (anthropologue du droit, Cirad), Olivier Barreteau (hydrologue, Cemagref), Stanislas Boissau (anthropologue et économiste, Cirad-Université Wageningen), François Bousquet (modélisateur, Cirad), William's Daré (sociologue, Cirad), Michel Étienne (écologue, Inra), Christophe Le Page (modélisateur, Cirad), Raphaël Mathevet (géographe, Tour du Valat), Guy Trébuil (agronome et géographe, Cirad), Jacques Weber (économiste et anthropologue, Cirad).
}

donc choisi de nous donner une doctrine réfutable ${ }^{1}$ sous la forme d'une «charte », pouvant être soumise à évaluation. Ce qui signifie :

- considérer que toutes les idées à la base de la modélisation ont comme vocation d'être remises en cause au contact du terrain, c'est-à-dire d'être volontairement et directement soumises à réfutation ;

- n'avoir aucune hypothèse implicite dans l'expérimentation : ceci constitue un objectif en soi de la démarche et implique le développement de procédures de mise en évidence de telles hypothèses à la fois sur le plan empirique et conceptuel, ce dernier plan consistant à confronter le modèle à des résultats reconnus par la communauté scientifique ;

- prendre en considération, dès les premières étapes, l'impact du processus de recherche sur le terrain, que ce soit en termes d'objectifs définis, de qualité de la démarche, d'indicateurs quantifiables, de suivi ou d'évaluation ;

- donner une attention particulière aux processus de validation de cette démarche de recherche, sachant qu'il n'existe pas de théorie générale de la validation des modèles et que des procédures autres que celles utilisées dans les modèles bio-physicomathématiques sont à envisager.

Ce document est le fruit d'une longue discussion et de nombreux travaux communs, au cours des cinq dernières années, entre les différents signataires. Cette charte est par définition évolutive, la volonté de rigueur nécessitant de l'améliorer à chaque étape et l'exigence de réfutation, de se remettre continuellement en question. Nous la présentons ici dans sa nouvelle version française («Charte $2.0 »)$.

${ }^{1}$ La réfutation est le seul moyen rigoureux de la liberté intellectuelle d'une recherche, particulièrement dans le contexte ambigu de la recherche-action. 


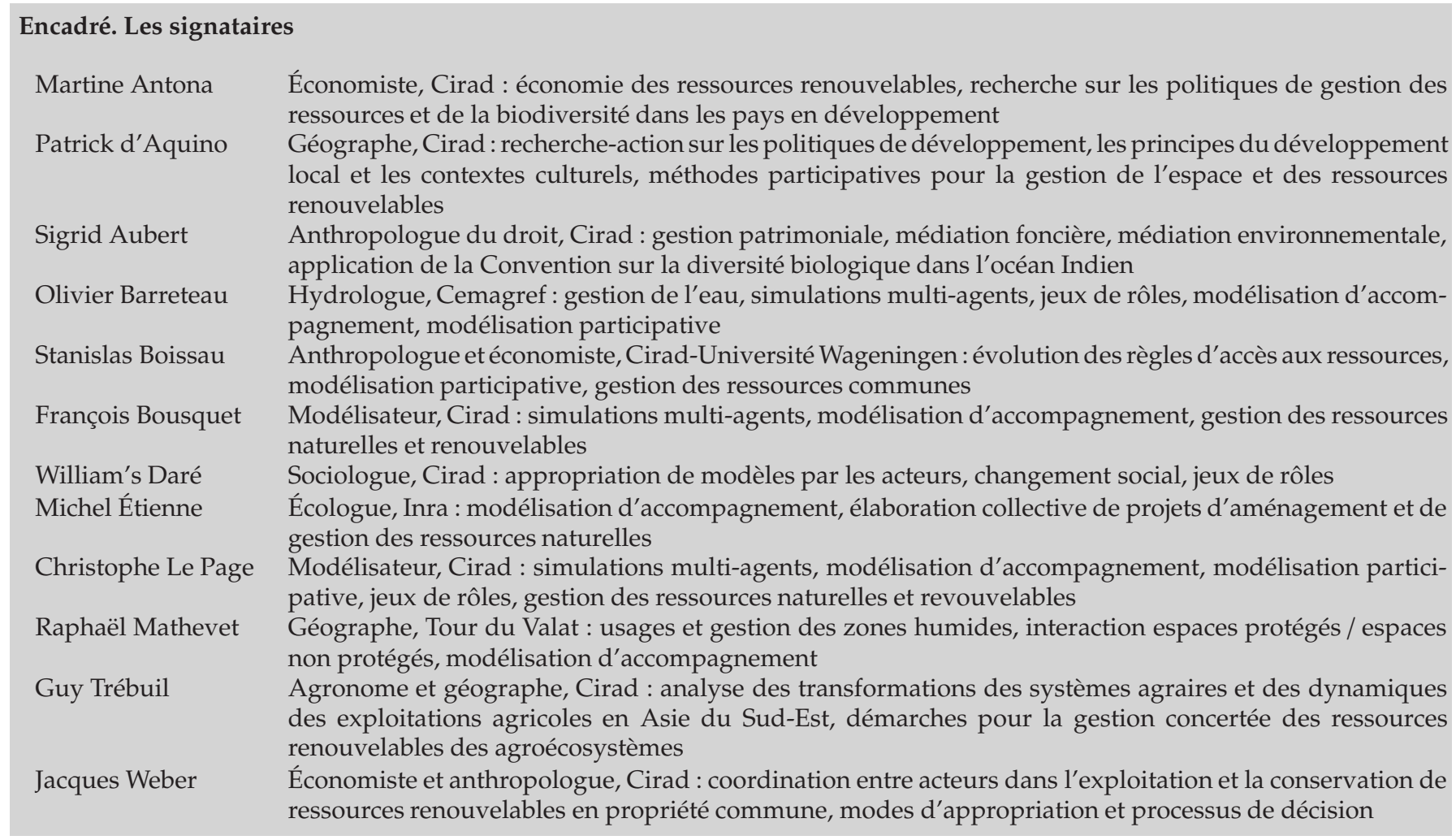

\section{La démarche commune}

En concordance avec le caractère dynamique et complexe des processus étudiés, notre démarche exige une confrontation continue et itérative entre théories et terrains, au travers de constants va-et-vient. Nous avons choisi de l'identifier sous le terme de modélisation d'accompagnement, ou companion modelling. Cette démarche est ainsi adaptée à la complexité et à l'ouverture des systèmes étudiés, car : 1 ) elle accepte comme légitimes et prend en compte des points de vue éventuellement contradictoires; 2) elle organise une remise en cause obligatoire de chaque nouvel élément introduit dans la démarche; 3) elle se confronte, à chaque cycle, à des éléments extérieurs nouveaux.

Étant donné que dans le domaine des sciences cognitives et de l'informatique, les systèmes multi-agents $(\mathrm{SMA})^{2}$ sont des outils particulièrement adaptés à la représentation de systèmes dynamiques et complexes et à l'exploration d'hypothèses présentées comme "vraies ",

\footnotetext{
${ }^{2}$ Modèles généralement informatiques capables de représenter un ensemble d'entités autonomes, situées dans un environnement, en interactions entre elles et avec celui-ci grâce à des capacités de perception, de représentation et d'action. Ils permettent d'analyser le système en termes d'agents qui ont différents points de vue, décident et agissent différemment, interagissent entre eux et avec leur environnement, et font partie d'organisations. Les SMA mettent en interaction tous ces éléments, ce qui permet de simuler des dynamiques au niveau du système.
}

cet outil de simulation a été privilégié dans notre démarche, notamment en association avec les SIG ou les jeux de rôles. Véritable géomatique déductive, les SMA contrebalancent la géomatique de type SIG par un travail déterminé par la formalisation des dynamiques et des hypothèses étudiées.

L'importance accordée dans notre démarche à la référence aux terrains implique qu'elle ait des retombées tangibles sur eux. Selon les expériences et les outils mis en place, ses apports peuvent être de trois types : 1) la modification des perceptions des acteurs ; 2) la modification de leurs façons d'interagir ; 3) la modification des actions qu'ils entreprennent.

Enfin, la distinction doit être faite entre deux contextes d'usage spécifiques et constitutifs de notre démarche : la production de connaissances sur des systèmes complexes et l'accompagnement de processus collectifs de décision. Alors que la première orientation correspond à de la recherche sur des systèmes via une forme particulière de relation au terrain, la seconde correspond à de la recherche sur des méthodes visant à faciliter la gestion concertée de ces systèmes.

\section{La connaissance des environnements complexes}

Cette conception et cet usage de la modélisation sont fondés sur - et invitent à approfondir - la relation 
dialectique existant entre chercheur, modèle et terrain. L'apport de la simulation y est envisagé comme l'accompagnement d'un processus itératif de recherche, spécifique à chaque situation. Le cycle continu terrain $\rightarrow$ modélisation $\rightarrow$ simulation $\rightarrow$ terrain rend compte du processus qui organise la démarche. Cela implique l'acceptation d'une diversité de modèles et de méthodes, œuvrant cependant tous à une nouvelle forme de relations entre la simulation, l'itinéraire de recherche et le processus de décision.

Le chercheur débute avec un premier modèle, qui a la vertu de le contraindre à expliciter ses préconceptions tant théoriques que vis-à-vis du terrain. Puis, la confrontation à la réalité lui fait réviser et reconstruire ce premier modèle, en prenant progressivement mieux en compte non seulement le terrain, mais aussi les questions que se posent les acteurs. La discussion des hypothèses du modèle et des simulations effectuées selon un plan d'expériences correspondant à des questions posées au préalable permet de modifier les premières questions posées et d'en formuler de nouvelles. Cette confrontation entre les dynamiques simulées par le modèle et celles observées sur le terrain conduit à la constitution d'un nouveau modèle, qui peut être une simple variante du premier ou un modèle entièrement nouveau. Le cycle se poursuivant, on arrive à une famille de modèles, trace des interactions successives entre modélisateur et terrain. Il n'y a en aucun cas, a priori, complexification progressive d'un modèle qui devrait incorporer de plus en plus d'éléments pour mieux coller à « la réalité ».

La famille de modèles ainsi constituée est un véritable système à base de connaissances qui permet au chercheur et aux acteurs ayant été en interaction d'accroître leurs connaissances personnelles et communes sur le système, sur les processus en cours et sur la place de chaque acteurobservateur dans le processus d'apprentissage collectif. L'enjeu primordial de la démarche d'accompagnement est alors une meilleure connaissance de ces processus plutôt qu'un itinéraire de gestion « clef en main » des ressources renouvelables. Cela se traduit par une conception particulière de la relation du modèle au terrain : ce n'est pas une simplification de la connaissance des acteurs qui est recherchée à travers lui, mais un outil facilitant la reconnaissance mutuelle par l'ensemble des acteurs de la représentation que chacun d'eux a de la question étudiée. Cette reconnaissance mutuelle s'appuie sur des indicateurs construits progressivement, en commun, au cours de la démarche et qui constituent les fondements de la modélisation d'accompagnement.

Tous les types de dialogues possibles entre chercheur et acteur sont considérés ici comme des processus de modélisation participative plus ou moins formalisés, depuis le diagnostic uniquement verbal jusqu'aux modèles informatiques. Dans tous les cas, il s'agit en effet de construire une représentation, de la partager puis de la formaliser sous une forme particulière : modélisations « inconscientes » (diagnostics participatifs uniquement verbaux, représentations cognitives d'un expert sur une situation...) et modélisations plus explicites (cartes, systèmes d'information, modèle informatisé...).

L'hypothèse sous-jacente est que, dans la plupart des situations de gestion de ressources renouvelables, ce n'est pas d'une simple formalisation de sa propre perception du problème que chaque acteur a besoin, mais d'un échange avec les autres acteurs (expert compris) sur les différentes perceptions en présence et les connaissances existantes. La simulation, en structurant ces échanges, permet aux acteurs de parvenir à valider les interactions entre les différentes représentations et les dynamiques du système intégrées dans le modèle. La simulation participe alors à un processus d'apprentissage sur le système étudié dans l'interaction avec et entre les acteurs locaux.

\section{L'accompagnement des processus collectifs de décision en situation complexe}

La «modélisation d'accompagnement » est une démarche permettant de faciliter des processus collectifs de décision, en proposant un travail d'explicitation des points de vue et des critères subjectifs auxquels se réfèrent implicitement, voire inconsciemment, les différentes parties prenantes. En effet, face à une situation complexe, le processus de décision est évolutif, itératif et continu. Cela signifie qu'il produit des actes de "décision » toujours imparfaits, mais dont le but est d'être, à chaque itération, mieux compris et plus partagés. Autrement dit, ce qui est recherché n'est pas dans la qualité du choix mais dans la qualité du processus qui y a conduit. Il ne s'agit pas de trouver la meilleure solution, mais de se donner les moyens de prendre en charge au mieux les incertitudes de la situation examinée en commun. Pour améliorer la qualité des processus collectifs de décision, la démarche cherche à rendre plus clairs et à faire partager les points de vue qui ont conduit à la situation étudiée. Elle se réfère à une perception dynamique du processus de décision, dans laquelle, en particulier, la perception scientifico-technique représente une simple option parmi d'autres dans le processus de prise de décision, et non pas une perception supposée juste vers laquelle doit tendre la décision. L'objectif n'est donc pas celui, fort ambitieux, de produire des décisions et des résultats définitifs, mais celui d'enrichir le processus de prise de décision, que ce soit sous son aspect technique (informations, qualité technique des actions entreprises...) ou sous son aspect sociologique (plus grande concertation, renforcement $\mathrm{du}$ pouvoir de l'acteur dans la décision...). Face à un processus évolutif, itératif et continu, il s'agit de proposer un accompagnement évolutif, itératif et continu. 
Quels outils peuvent prendre part à ce processus et comment les utiliser pour accompagner la dynamique collective de décision, c'est-à-dire pour aider les acteurs à gouverner au fil d'un itinéraire continu et à travers un processus d'enrichissement progressif, au lieu de proposer des solutions d'expertise? Nous sommes ici dans le cadre d'une approche expérimentale de la science " postnormale » où, à partir d'une conception partagée sur l'évolution de la situation présente, les acteurs peuvent s'engager ensemble dans un processus de prise en charge de l'incertitude. C'est ainsi que nous envisageons l'utilisation combinée de différents supports d'accompagnement au processus de décision (SMA, jeux de rôles, SIG, SGBD, audit patrimonial, outils de régulation économique ou juridique...). Selon les situations, la production de connaissances ou de points de vue sur un système donné pourra se traduire par :

- une amélioration de la connaissance des acteursdécideurs;

- une facilitation de la concertation entre acteurs (expert compris), au moyen d'un cadre de discussion et de partage de l'information, un échange de points de vue, de connaissances, de croyances entre acteurs, ou

- une aide à la négociation, visant ici un rapprochement de points de vue divergents dans une situation de conflit donnée.

La modélisation d'accompagnement s'insère alors dans un processus de médiation, même si elle ne couvre pas à elle seule l'ensemble du processus. Les acteurs apprennent collectivement en créant, modifiant ou observant les simulations. Car simuler, c'est agir sur le processus de décision, en créant ou modifiant des représentations. La modélisation d'accompagnement conduit les acteurs à partager des représentations et des simulations, comprenant les actions possibles (règles, aménagement...) qu'ils envisagent sur le milieu. Cependant, elle ne prend pas en charge les autres étapes possibles du processus, qui concerneraient une expertise plus quantifiée (taille d'un aménagement, production estimée, etc.). Elle intervient donc en amont de la décision technique, lorsqu'il s'agit d'appuyer la réflexion des différents acteurs concernés, en vue de parvenir à une représentation partagée de la problématique et des voies possibles pour engager un processus de prise en charge.

\section{Une utilisation conjointe}

Nous considérons que l'organisation de l'action est le résultat qui émerge d'une dynamique d'interactions entre acteurs, individuels et/ou collectifs. Cette dynamique est elle-même contrainte par la compréhension, la perception, que chaque acteur a de ses actions et des actions des autres. Et les modalités d'interaction vont fortement dépendre des indicateurs mobilisés pour évaluer l'environnement que chaque acteur partage avec les autres. Il est donc important de distinguer rigoureusement les deux voies d'application de notre démarche, même si elles sont souvent empruntées simultanément dans la pratique. La première voie cherche sa légitimité scientifique dans la production de connaissances et la pertinence de celles-ci, la deuxième voie cherche sa légitimité scientifique dans l'amélioration de la qualité des processus de décision collective.

Dans les deux cas, il y a production de connaissances. Et cette production de connaissances est spécifique puisqu'elle a lieu dans le cadre d'une interaction entre des chercheurs et des acteurs locaux. Mais, dans le premier cas, elle est l'objectif (qu'elle soit à destination des chercheurs ou des acteurs locaux dans des actions de formation), alors que, dans le second cas, on fait l'hypothèse que c'est un élément de méthode nécessaire pour atteindre un objectif principal qui est l'appui à la décision collective. Cette distinction concerne autant la question méthodologique que les plans épistémologique et analytique : rien ne dit que les outils et les méthodes éprouvés dans un cas seront utiles, efficaces et adaptés dans l'autre, en particulier en ce qui concerne la position du chercheur/modélisateur dans le processus. C'est pourquoi nous abordons de façon différente mais conjointement ces deux problématiques de modélisation.

Cette charte reste une première étape de réflexion portée par un collectif de chercheurs confrontés à une forte interrogation sur les possibilités, conscientes ou non, de manipulation des processus de décision par l'utilisation de modèles pouvant conduire à des situations non voulues par les acteurs. Elle érige en règle déontologique la nécessité d'implication du chercheur dans le processus d'accompagnement, la transparence des hypothèses et des procédures sous-jacentes, un affichage clair des domaines d'utilisation des modèles développés et la remise en cause continue de la démarche proposée. 